\title{
How Do Weekly Magazines Provide Information on Urogenital Cancer to the Public in Aged Societies?
}

\author{
Masayoshi Nagata $^{1^{*}}$, Tomoko Matsumura², Masahiro Kami ${ }^{2}$ \\ ${ }^{1}$ Department of Urology, National Center of Global Health and Medicine, Tokyo, Japan \\ ${ }^{2}$ Division of Social Communication System for Advanced Clinical Research, \\ The Institute of Medical Science, The University of Tokyo, Tokyo, Japan \\ Email: *yql02037@nifty.com
}

Received September 2, 2013; revised October 1, 2013; accepted October 9, 2013

Copyright (C) 2013 Masayoshi Nagata et al. This is an open access article distributed under the Creative Commons Attribution License, which permits unrestricted use, distribution, and reproduction in any medium, provided the original work is properly cited.

\begin{abstract}
Little information on cancer coverage rather than newspaper and television is available. Japanese weekly magazines have a circulation of over 2,700,000 per week. To examine how they delivered urogenital cancer information to the public, cancer-related articles and advertisements in six major Japanese weekly magazines from 2009 to 2010 was analyzed. $1.8 \%$ of total articles and advertisements were cancer-related. Prostate cancer $(n=119)$ was the second-most common topic, following lung cancer $(\mathrm{n}=145)$, whereas only three articles were published on kidney or bladder cancer. The 53 articles on therapies for prostate cancer comprised radiotherapy $(n=29)$, surgery $(n=16)$, chemotherapy $(n=4)$, and others $(\mathrm{n}=4)$. All 42 comments or interviews were cited in the article on prostate cancer, while 26 of them were attributed to only two famous doctors. Although cancer coverage in weekly magazines could be useful to spread information on prostate cancer, we should recognize their considerable bias based on a disproportionate emphasis.
\end{abstract}

Keywords: Cancer Coverage; Weekly Magazine; Prostate Cancer

\section{Introduction}

Cancer is one of the major concerns in aged societies. Some researchers investigated cancer coverage on media, while most reports were related to newspapers [1,2] or television [3]. Little information is available on the other media.

Weekly magazines have the second-largest circulation following newspapers in Japan and provide broad information for a large readership, particularly focusing on middle-aged males. The Japanese weekly magazines could be thought as equivalent to the tabloids in Western countries. In the previous report, we demonstrated that cancer articles in weekly magazines were common paper media for providing cancer information to the public, although the information provided might place emphasis on unestablished treatments or biased opinions [4]. After a famous Japanese comedian, Mr. Kampei Hazama, confessed prostate cancer, numbers of prostate cancer articles increased over three-fold (2.0 to 6.6) [4]. In this study, we investigated cancer-related articles and advertisements on weekly magazines, particularly on urogenital cancer, to demonstrate trends in the public in aged

\footnotetext{
*Corresponding author.
}

societies.

\section{Materials and Methods}

Cancer-related articles and advertisements in six major Japanese weekly magazines (Weekly Gendai, Sunday Mainichi, Weekly Bunshun, Weekly Asahi, Weekly Shincho, and Weekly Post) with the circulation of approximately 2,700,000 per week, were investigated from July 2009 to December 2010. All articles including urogenital cancer were extracted and classified according to types of primary sites, topics, and treatment. Comments, interviews, and serial columns from oncologists cited in cancer-articles were examined to evaluate their adequateness by two authors (M. N. and M. K.).

\section{Results}

We extracted 1037 (1.8\%) cancer-related articles and advertisements in total 58,632. Prostate cancer $(n=119)$ was the second-most common topic, following lung cancer $(n=145)$. Only three articles were published on kidney or bladder cancer. Topics of the 119 articles on prostate cancer comprised therapies $(n=53)$, case reports $(n=28)$, checkup of prostate-specific antigen (PSA) $(n=8)$, and 
others $(\mathrm{n}=20)$. The 53 articles on therapies comprised radiotherapy $(n=29)$, surgery $(n=16)$, chemotherapy ( $n$ $=4)$, and others $(n=4)$. Many celebrities disclosed acquiring prostate cancer. Whereas number of prostate cancer articles increased after publishing Mr. Hazama's prostate cancer story, advertisements of an enlightenment activity which advocate PSA checkup, "Blue Clover Campaign", appeared in magazines during the periods. All 42 comments were cited in the 119 article on prostate cancer, while 26 of them were attributed to two famous doctors (a radiologist and an urologist).

\section{Discussions}

Magazines are useful media for providing information on urogenital cancer. They could be an effective measure that assists the enlightenment of prevention and early detection of prostate cancer, since a large part of the readership is aged males. However, they have issues to be discussed. Firstly, prostate cancer was the topic in 97\% of all the articles on urogenital cancer. It should be noted that morbidities of prostate, bladder and kidney cancer in Japanese males are 69, 20, and 16 per 100,000 respectively. Magazines are insufficient for delivering information on bladder or kidney cancer. It would be of interest if readers in general might be more interested in prostate cancer as more people are affected and hence, magazines just mirror that interest. Alternatively it might as well be possible that magazines are motivated to create an interest.

Secondly, celebrities with cancer probably play a pivotal role in drawing publics' attention to cancer. While most cancer coverage in newspaper is related to politics and incidents [1], those in magazines are described through the eyes of each patient. Interestingly, numbers of cancer-related articles increased following confession of affected celebrities. These facts are comparable to previous reports in the Western countries [5,6].

Third, considerable bias exists in cancer-articles. Surgery is a major treatment option for prostate cancer in Japan [7]. More than half of prostate cancer articles were, however, on radiotherapy because a famous radiologist was in charge of serial column. Surprisingly, $62 \%$ of the comments were made by only two doctors. Journalists hope to use comments of big-name specialists, which attract attention from readers. Although each comment was medically pertinent, these situations might easily cause prejudicial understanding in readers.

Finally, there are some limitations in this study to be discussed. The observation periods were too short to draw a definite conclusion. Further long-term studies are required. Next, these articles could be used as platforms for open or hidden advertisements. Consequently, the number and potential influence of cancer-related advertisements were probably underestimated, since any investment or finance to the magazines by hospitals, office urologists, or pharmaceutical companies might affect their contents. Lastly, there could be a problem with the assumption that these six "Japanese" magazines could represent other innumerable magazines. We need to extend over a wide range of magazines, including online magazines and other countries' magazines.

While cancer coverage in weekly magazines is useful to spread information on prostate cancer around the public, we should recognize their distinctive characteristics

\section{REFERENCES}

[1] Y. Kishi, S. Nagamatsu, M. Takita, Y. Kodama, A. Hori, N. Hatanaka, T. Hamaki, E. Kusumi, K. Kobayashi, T. Matsumura, K. Yuji, H. Narimatsu, Y. Tanaka and M. Kami, "Trends in Cancer Coverage in Japanese Newspapers,” Journal of Clinical Oncology, Vol. 26, No. 36, 2008, pp. 6017-6020.

http://dx.doi.org/10.1200/JCO.2008.20.1392

[2] I. H. Jones, J. M. Williamson and D. B. Hocken, "How Informative Is the Print Media Coverage of Colorectal Cancer?” Colorectal Disease, Vol. 14, No. 2, 2012, pp. 250-252. http://dx.doi.org/10.1111/j.1463-1318.2011.02607.x

[3] J. Niederdeppe, E. F. Fowler, K. Goldstein and J. Pribble, "Does Local Television News Coverage Cultivate Fatalistic Beliefs about Cancer Prevention?” Journal of Communications, Vol. 60, No. 2, 2010, pp. 230-253. http://dx.doi.org/10.1111/j.1460-2466.2009.01474.x

[4] M. Nagata, M. Takita, Y. Kishi, Y. Kodama, T. Matsumura, N. Murashige, Y. Homma and M. Kami, "Cancer Articles in Weekly Magazines: Useful Media to Deliver Cancer Information to the Public?” Japanese Journal of Clinical Oncology, Vol. 43, No. 4, 2013, pp. 426-430. http://dx.doi.org/10.1093/jico/hyt004

[5] D. Metcalfe, C. Price and J. Powell, "Media Coverage and Public Reaction to a Celebrity Cancer Diagnosis," Journal of Public Health, Vol. 33, No. 1, 2011, pp. 80-85. http://dx.doi.org/10.1093/pubmed/fdq052

[6] S. Pillet, "Celebrities May Help Bring Positive Media Attention to Cancer,” ONS Connect, Vol. 24, No. 9, 2009, p. 16.

[7] H. Fujimoto, H. Nakanishi, T. Miki, Y. Kubota, S. Takahashi, K. Suzuki, H. O. Kanayama, K. Mikami and Y. Homma, "Oncological Outcomes of the Prostate Cancer Patients Registered in 2004: Report from the Cancer Registration Committee of the JUA,” International Journal of Urology, Vol. 18, No. 12, 2011, pp. 876-881. http://dx.doi.org/10.1111/j.1442-2042.2011.02895.x 